\title{
ESCUELA COMO FÁBRICA DE SUJETOS. GÉNESIS DE LA MODERNIDAD ESCOLAR EN MÉXICO
}

\author{
ESCOLA COMO FÁBRICA DE SUJEITOS. \\ GÊNESE DA MODERNIDADE ESCOLAR NO MÉXICO
}

\author{
THE SCHOOL AS A FACTORY OF SUBJECTS. \\ GENESIS OF SCHOLAR MODERNITY IN MEXICO
}

Ana María Valle Vázquez ; Marco Antonio Jiménez García²

\begin{abstract}
Resumen
Se trata de un artículo que describe cuatro formas en las cuales se evidencian los ejercicios de poder como producción de sujetos, a saber: la guerra, la lucha de razas, la disciplina y la normalización, esto se muestra con prácticas escolares concretas del México moderno. El método utilizado problematiza la escuela como fábrica de sujetos, empleando herramientas conceptuales foucaultianas. Aceptamos, con Foucault, que no se trata de preguntar a los sujetos cómo, por qué y en nombre de qué derecho (divino, económico o jurídico) pueden aceptar o dejarse someter, sino que se pretende mostrar cómo las relaciones de sometimiento concretas fabrican a los sujetos. En los ejercicios de poder no hay sujeto neutral, ya que siempre se es forzosamente el adversario de alguien. Uno de los resultados de este ensayo es que el maestro es un sujeto de los ejercicios de poder de la enseñanza escolar básica, donde las relaciones de poder a las que se ha sometido el normalista son la distinción racial, la normalización de saberes y el trabajo medicalizado al servicio del Estado. Estas relaciones de fuerza de la vida y sobre la vida son las formas como hemos defendido la sociedad moderna a la cual pertenecemos.
\end{abstract}

Palabras clave: escuela moderna, fábrica de sujetos, ejercicios de poder, disciplina, normalización.

\section{Resumo}

Trata-se de um artigo que descreve quatro maneiras nas cuales são evidenciados os exercícios de poder como produção de sujeitos, a saber: a guerra, a luta racial, a disciplina e normalização, isto é mostrado com práticas escolares específicas do México moderno. O método utilizado problematiza a escola como fábrica de sujeitos, usando ferramentas conceituais foucaultianas. Aceitamos, com Foucault, que não trata-se de perguntar aos sujeitos como, por que e em nome de que direito (divino, econômico ou legal) podem aceitar ou deixar someter, mas destina-se a mostrar como as relações de sujeição específicas fabrican aos sujeitos. No exercício do poder nenhum sujeito é neutro, já que é necessariamente sempre o adversário de alguém. Um resultado deste teste é que o professor é um sujeito do exercício do poder da educação escolar básica, onde as relações de poder às que foi submetido o normalista são a distinção racial, a padronização de

\footnotetext{
${ }^{1}$ Doctora en Pedagogía - Facultad de Filosofía y Letras - Universidad Nacional Autónoma de México (UNAM), Cidade do México, México. Profesora en el Centro de Investigación y Docencia en Humanidades del Estado de Morelos. Docente del posgrado - Universidad Nacional Autónoma de México (UNAM) - Cidade do México, México. E-mail: anvallev@gmail.com

2 Doctor en Ciencias Sociales - por la Universidad Autónoma Metropolitana Unidad Xochimilco (UAM-

Xochimilco), Cidade do México, México. Profesor titular de la FES-Acatlán UNAM.E-mail: marcoacatlan@gmail.com
}

(C) ETD-Educação Temática Digital $\quad$ Campinas, SP $\quad$ v.19 $\quad$ n.4 $\quad$ p.642-668 $\quad$ out./dez. 2017 
conhecimento e trabalho medicalizada ao serviço do Estado. Estas relações de força da vida e em a vida são as maneiras como temos defendido a sociedade moderna a que pertencemos.

Palavras chave: Escola moderna, Fábrica de sujeitos, Exercícios do poder, Disciplina, Normalização.

\begin{abstract}
Fabrication of subjects, comes to light: in war, in race struggles and through discipline and normalization. All of the above is shown through an analysis of specific school practices in modern Mexico. The methodology used in this text problematizes the term "school", as a factory of subjects, with the help of terminology used by Foucault.Just like Foucault, we argue that it is not about asking subjects how, why and in the name of which specific right (divine, economic or legal) they can accept or let themselves be dominated but about how subjects are fabricated through specific relations of domination. In the exercises of power there is no neutral subject because we are always and forcefully somebody's adversary. In this essay, we argue that the teacher is the subject of the exercises of power that occur within primary education. In this sphere, the primary school teacher and Normal school graduate submits to power relations such as: racial distinction, the normalization of knowledge and the medical work that is at the service of the state. This relations of power in life and about life are the ways in which we have defended the modern society we live in.
\end{abstract}

Key words: Modern school, Fabrication of subjects, Exercise of power, Discipline, Normalization.

En vez de preguntar a unos sujetos ideales qué cedieron de sí mismos o de sus poderes para dejarse someter, es preciso investigar la manera en que las relaciones de sometimiento pueden fabricar sujetos Foucault

\title{
PRESENTACIÓN
}

El título Defender la sociedad es una provocación que Foucault hace para pensar, desde el estado de guerra y las relaciones de poder como raza, nación y seguridad, el "bien" de "nuestra comunidad", una comunidad a la que "hay que defender" y "hacer vivir". Cuando pensamos en la comunidad escolar y sus ejercicios de poder ¿qué escuela defendemos? Los ejercicios de poder son constitutivos de toda sociedad y la escuela no escapa a estos ejercicios que fabrican sujetos, porque el poder trata de las fuerzas ocurridas en los vínculos al interior del cuerpo social y de sus instituciones. En la escuela, la dominación no sólo es la que ejerce el director sobre maestros y alumnos, o el docente sobre los indefensos estudiantes, sino que el control está en las relaciones recíprocas entre alumnos, docente, autoridad etcétera.

En el presente artículo se pretende describir cuatro formas en las cuales se evidencian los ejercicios de poder como producción de sujetos, tales como: la guerra, la lucha de razas, la disciplina y la normalización. Dichos ejercicios Foucault los ubican desde la segunda mitad del siglo XVIII y corresponden a lo que en Europa se llamó la época clásica. Lo que aquí intentamos es ilustrar con prácticas escolares concretas estas cuatro formas de ejercicios de poder que fabrican sujetos. Para ello recurriremos principalmente a experiencias escolares de México. Es interesante señalar que una distinción sustantiva

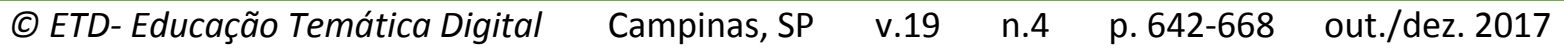


de la época clásica es la relación que existe entre guerra, raza y pureza, donde las pretensiones de higiene al interior de la sociedad serán determinantes para la vida individual y colectiva. La escuela será un lugar privilegiado para cumplir estos objetivos de purificación social.

La escuela como fábrica de sujetos puede analizarse con Foucault desde diversos ángulos, uno de ellos es a partir de la episteme y otro puede ser desde el biopoder. Sin negar la importancia que una y el otro tienen en el pensamiento de Foucault, en este escrito nos centraremos en algunas formas de biopoder y biopolítica a través de la disciplina y la normalización. Entendemos por biopoder al control, gobierno o sometimiento de la vida humana, en todas sus dimensiones, desde el cuerpo individual hasta la sociedad en su conjunto. Es decir el biopoder se encarna en la disciplina como control del cuerpo y en la biopolítica como el control de las poblaciones modernas e ilustradas. Lo que Foucault muestra en eso que llamó "ejercicios de poder" no es otra cosa que formas de control y dominio de la vida, que su vez permiten y potencian la vida misma.

La pregunta ¿cómo las relaciones de sometimiento fabrican a los sujetos?, es un cuestionamiento por el control que posibilita la vida. Pensar la escuela como fábrica de sujetos implica identificar los ejercicios de poder que producen vida en la escuela. Consideramos que las nociones de guerra, lucha de razas, disciplina y normalización son fuerzas que se manifiestan en las prácticas escolares y esto permite que germine y se cristalice la vida individual y social.

El texto está dividido en dos apartados, en el primero describimos las nociones de: ejercicios de poder, guerra y raza como fundamentos de la fábrica de sujetos; y en el segundo apartado revisamos algunas prácticas escolares mexicanas que dan cuenta de la defensa de las sociedades disciplinarias y de normalización.

\section{EJERCICIOS DE PODER Y FÁBRICA DE SUJETOS}

Ejercicios de poder

De acuerdo con Foucault "el poder no se da, ni se intercambia, ni se retoma, sino que se ejerce y sólo existe en acto. Contamos, igualmente, con otra afirmación: la que el poder no es en primer término, mantenimiento y prórroga de las relaciones económicas, sino, primariamente una relación de fuerzas en sí mismo" (FOUCAULT, 2014, p. 27) El poder lo que quiere es mantenerse a sí mismo para sostener la vida. Se trata del poder que se ejerce sobre la vida y simultáneamente del poder de la vida, no en un sentido abstracto sino en una condición histórica y social. El poder es tanto acción, ejercicio y

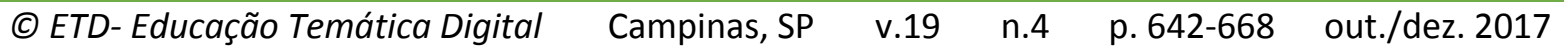


práctica, como relación de fuerza en sí mismo, es decir, se trata de un ejercicio de fuerza, es una práctica de resistencia y es una acción bélica.

Si aceptamos, como dice Foucault (2014, p. 28-29) que el poder reprime, que es una relación de fuerzas, entonces podemos decir que las prácticas y los saberes escolares, como ejercicios de poder, reprimen otras prácticas y saberes. No hay prácticas ni saberes inocentes, que no busquen imponerse o reprimir a otros. Dichos ejercicios de sometimiento o de relaciones de fuerza habidos en las prácticas y saberes escolares no son buenos ni malos, no son mejores o peores que otros, sólo son tensiones que permiten la producción de subjetividad. El poder, dice Foucault,

[...] obliga a producir la verdad, dado que la exige, la necesita para funcionar; tenemos que decir la verdad, estamos forzados, condenados a confesar la verdad o a encontrarla. El poder no cesa de cuestionar, de cuestionarnos; no cesa de investigar, de registrar; institucionaliza la búsqueda de la verdad, la profesionaliza, la recompensa. [...] somos juzgados, condenados, clasificados, obligados a cumplir tareas, destinados a cierta manera de vivir o a cierta manera de morir, en función de discursos verdaderos que llevan consigo efectos específicos de poder (FOUCAULT, 2014, p. 34)

Los ejercicios de poder habidos en la escuela institucionalizan la verdad y a partir de ella nos clasifican, nos juzgan, nos obligan a cumplir tareas, nos imponen una forma de vida y muerte. Por ejemplo, como veremos más adelante, en la distinción racial del magisterio propia del México virreinal, en la disciplina de la enseñanza mutua de Lancaster o en la escuela normal mexicana, se fundan y mantienen verdades como instrumentos de poder, como herramientas de guerra y política que permiten la permanencia de la sociedad a la cual pertenecen. Las palabras, las cosas y las costumbres que aprendemos en la escuela dan sentido a la sociedad a la cual pertenecemos.

La represión bélica y política de unos saberes sobre otros es inevitable, por lo que sería absurdo intentar eliminar, de una vez y para siempre, las diferentes formas de sometimiento, puesto que a diversas maneras de emancipación corresponderán diversas condiciones de control. El problema está cuando se banaliza la guerra y la política constitutivas de todo ejercicio de poder, de todo régimen de verdad, de toda producción de sujeto o de toda práctica educativa. Lo terrible no sólo es intentar eliminar prácticas y saberes "malos" para imponer otros "buenos", sino pretender quitar el sometimiento en sí mismo aparentando que "todos somos iguales", "que todos tenemos los mismos derechos", "que la escuela inclusiva es la que no excluye" cuando la diferencia, la asimetría y la exclusión son constitutivos de toda relación de fuerzas que mantienen la vida. 
Sin ejercicios de poder, sin regímenes de verdad y sin fabricación de sujetos no hay vida humana posible, sin guerra no existe política, sin represión no existen saberes y sin control no existen procesos de subjetivación. Considerar que la escuela puede eliminar el sometimiento de unos saberes y formas de existencia es no ver que la vida sólo es posible a partir de las relaciones de fuerzas. "Lo políticamente correcto", "la educación para la paz", "la igualdad y equidad para todos" tan anheladas en nuestros días, son apariencias que debilitan a la política, a la educación, a la amistad, a la solidaridad y a la identidad. Quien piense que la política elimina la guerra, que la amistad niega al enemigo, que la solidaridad y la identidad rechazan la diferencia y la exclusión, no ven la guerra en los engranajes de la paz. Por ejemplo, pensar que el diálogo escolar elimina el conflicto o que la inclusión de niños discapacitados en ambientes "normales" garantiza la equidad, son sólo apariencias que intentan ocultar los ejercicios de poder.

Foucault en Defender la Sociedad estudia el poder a partir de las técnicas de dominación, lo cual de ninguna manera significa que juzgue al poder y la represión como algo negativo o neutral, contra lo cual hay que luchar e intentar eliminar. El enemigo no es el poder, no es el sometimiento racial o de clase, ni la disciplina de los cuerpos y tampoco es la normalización. El enemigo en esta época, tal vez, es la incapacidad de ver al enemigo. Una de las raíces de esta incapacidad está en la idea de que la educación en general y la escuela en particular, pueden eliminar el control o sometimiento, es decir, pensar que la educación y la escuela nos hacen libres, buenos y mejores, sin darnos cuenta que incluso esta romántica posición es una forma de dominación. La dominación, aclara Foucault,

[...] no me refiero al hecho macizo de una dominación global de uno sobre los otros o de un grupo sobre otro, sino a las múltiples formas de dominación que pueden ejercerse dentro de la sociedad: en consecuencia, no al rey en su posición central, sino a los súbditos en sus relaciones recíprocas; no a la soberanía en su edificio único, sino a los múltiples sometimientos que se producen y funcionan dentro del cuerpo social (FOUCAULT, 2014, p. 36)

En la escuela, la dominación no sólo es la que ejerce el director sobre maestros y alumnos, o el docente sobre los "indefensos" estudiantes, mucho menos el Estado sobre la institución escolar, sino que la dominación incluso está en las relaciones entre alumnos o dentro del cuerpo docente. El poder trata de las fuerzas ocurridas en los vínculos al interior del cuerpo social y de sus instituciones. Dominaciones que van constituyendo sujetos.

Los procesos de subjetivación no son otra cosa que procesos de dominación, no hay sujeto libre ni neutral, exento de sometimiento, en todo caso la libertad es una forma de ejercicio de poder. Por ello no se trata de "preguntar a los sujetos cómo, por qué y en nombre de qué derecho (divino, económico o jurídico) pueden aceptar dejarse someter,

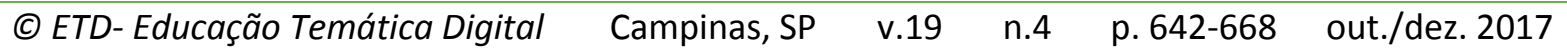


sino mostrar cómo los fabrican las relaciones de sometimiento concretas" (FOUCAULT, 2014, p. 50). El problema no es que los indefensos alumnos se dejen someter por los terribles maestros o que los pobres maestros sean dominados por las maléficas políticas educativas, esto no es el asunto a resolver, porque no hay igualdad ni simetría puras, siempre la diferencia y la asimetría están en los engranajes que fabrican a los sujetos en sus relaciones de sometimiento.

Frente a aquellos que han abjurado del carácter reproductor de la escuela y la han colocado del lado liberador, en un extremo, o por lo menos la consideran como una arena de lucha en donde se confrontan diferentes posiciones, en lo que refiere a la educación de niños no hay que olvidar que, más allá de una condición liberadora o de un especio de confrontación, de lo que se trata fundamentalmente es de garantizar la permanencia de la sociedad. Esta última cuestión va a transformarse conforme el sujeto se desarrolla y pasa de la infancia a la adultez. Sin embargo, el carácter fabricador de la educación pre-escolar (kindergarden) la educación media y superior no deja de estar presente.

Si en la aristocracia hay diferencias ¿por qué no habría de haberlas en el pueblo? Mantener las diferencias garantiza la sobrevivencia de toda institución, sociedad y cultura. Por ello, como veremos más adelante la distinción racial en el México virreinal fue un elemento fundamental para el ejercicio magisterial; o bien la identificación de las diferencias en las capacidades de los alumnos es sustantiva para la enseñanza mutua de Lancaster, tan bien acogida en las escuelas normales de México. Las prácticas y los saberes de y entre maestros, alumnos y directivos son modos de ejercer el poder, son formas de sometimiento y, por tanto, son procesos de subjetivación.

Nos parece que una característica de nuestra época globalizada es considerar como deseable y benéfico la igualdad, la equidad, la inclusión o la democracia, asumiendo que en ello no hay relaciones de sometimiento concretas. Dicho de otra manera con palabras de Foucault "se hace creer a los inferiores que un poco más de igualdad en su beneficio traerá más libertad para todos. Y de hecho, gracias a esa igualitarización, se desemboca en un gobierno despótico" (FOUCAULT, 2014, p. 139) La frase "todo por el pueblo, pero sin el pueblo" es el proyecto igualitarizador del despotismo. Todo proyecto educativo que pretenda igualdad o diga tener sus fundamentos en ella, es un proyecto déspota. La pregunta no es por el despotismo ni por los proyectos educativos déspotas sino ¿cómo el ejercicio de poder habido en la escuela fabrica subjetividad? Para intentar dar respuesta a esta pregunta revisemos tres nociones que destaca Foucault en los ejercicios de poder: la guerra, la política y la raza; y cómo éstos están en las prácticas escolares modernas. 


\section{Guerra, política y raza}

"La política es la continuación de la guerra por otros medios", dice Clausewitz (siglo XVIII), cuando en principio, como lo demuestra Foucault, siguiendo a Boulainvilliers (siglo XVII), la frase es: "la guerra es la continuación de la política por otros medios". La política no comienza cuando cesa la guerra, ni la guerra comienza cuando termina la política. Una y otra son formas de ejercicio de poder, son formas de dominación, son prácticas de relaciones recíprocas. Tanto la guerra como la política son estados o condiciones de vida social.

Sabemos que la ley jurídica y el derecho nacen de las masacres, de las devastaciones y de las conquistas, la ley y el derecho no son pacíficos, no destensan las relaciones entre sujetos y tampoco son laxantes sociales,

[...] la guerra es el motor de las instituciones y el orden: la paz hace sordamente la guerra hasta en el más mínimo de sus engranajes. En otras palabras, hay que descifrar la guerra debajo [al interior, en las entrañas] de la paz: aquella es la cifra misma de ésta. [...] No hay sujeto neutral. Siempre se es, forzosamente, el adversario de alguien (FOUCAULT, 2014, p. 56)

La escuela en todos sus niveles no escapa a este imperativo de la guerra ni de la política. Habría que buscar la barbarie en los engranajes de las prácticas escolares. Es un error considerar que la llamada educación para la paz es algo bueno, que nos hace más inclusivos, equitativos y tolerantes, sin aceptar que toda educación, como proceso de humanización, conlleva actos beligerantes; y que toda inclusión es una forma de exclusión, que la equidad siempre hace evidente las diferencias y que la tolerancia es un reservorio de odio y crueldad que puede estallar en cualquier momento. La educación para la paz es la cifra misma de la educación para la guerra.

Si no hay sujeto neutral podemos decir que tampoco hay institución imparcial. Los ministerios de educación, las Naciones Unidas, la UNESCO, los Derechos Humanos no son inmunes a la guerra, al poder o a la tensión habida en las relaciones entre sujetos. En tal sentido la escuela, como toda institución educativa, es una institución de conflicto, es el lugar donde se practica, se aprende y se ejercen las relaciones de dominación y sometimiento constitutivos de toda relación humana. La diferencia consiste en que en la escuela hay disciplina y normas que deben ser funcionales para el conjunto de relaciones sociales. Por ejemplo, otra institución que educa es la familia y ésta tiene sus propia disciplina y normas que pueden ser compartidas en la escuela pero que no necesariamente son las mismas. 
Cuando Foucault dice que no hay sujeto neutral y que siempre se es, forzosamente, el adversario de alguien nos lleva al imperativo que está en el título de su curso de 1976: Hay que defender la sociedad. El mandato "hay que" significa que no hay sujeto ni institución neutral y tampoco hay ejercicio de poder imparcial. La condición de sujeto nos hace ser siempre adversario de alguien y conlleva la tensión entre fuerzas de subjetivación. Pero ¿qué significa defender? y ¿a qué sociedad se defiende? Hasta aquí podemos decir que "defender" significa hacer la guerra o, mejor dicho, asumir el estado de guerra en el que como seres humanos vivimos; la guerra como motor de la producción de subjetividad, la guerra como tensión inevitable que hay en todo ejercicio de poder. Y la "sociedad" es la noción que da sentido al grupo o a la comunidad a la cual se pertenece; Foucault identifica las nociones de raza, nación y seguridad para analizar eso que hemos llamado sociedad, desde el siglo XVII, y a lo cual hay que defender. Para defender la sociedad hay que buscar defender las instituciones que la conforman, como: la escuela, los hospitales, la iglesia, la familia, el ejército, los partidos políticos, las empresas, las fábricas, etcétera. De ninguna manera se trata de un "defender la sociedad" en abstracto, sino a partir de los sujetos, razas, grupos y colectivos que se organizan, voluntaria o artificialmente, en instituciones particulares. Por eso "defender la escuela" es un derivado obligado del imperativo "hay que defender la sociedad".

Ahora pasemos a la relación entre guerra y raza. El estado de guerra, ineludible para toda sociedad, es el enfrentamiento cotidiano y normal de todos entre o contra todos, es la condición de vida social que provoca el conflicto diario de unos contra otros. Cuando Foucault habla de la guerra no se refiere exclusivamente a aquella que se efectúa en un campo de batalla con armas y donde hay muertos, heridos, vencidos y vencedores, sino fundamentalmente Foucault se refiere al combate diario que se establece en cualquier situación. La guerra no sólo es la armada, antes bien refiere a las relaciones de fuerza que tenemos con los vecinos de nuestra casa, con los colegas, con los alumnos, con los familiares, con los amigos, con los desconocidos en la vía pública, etcétera. Es la condición o el estado en el cual nos relacionamos con los otros al interior de la sociedad a la cual pertenecemos. No es la guerra de los de adentro contra los de afuera, antes bien se trata de la lucha al interior de todo tejido social,

[...] la otra raza, en el fondo, no es la que vino de otra parte, la que triunfó y dominó por un tiempo, sino la que se infiltra permanentemente y sin descanso en el cuerpo social o, mejor, se recrea constantemente en el tejido social y a partir de él. En otras palabras: lo que vemos como polaridad, como ruptura binaria en la sociedad no es enfrentamiento de dos razas recíprocamente exteriores, es el desdoblamiento de una única raza en una superraza y una subraza. O bien, la reaparición, a partir de una raza, de su propio pasado. En síntesis, el reverso y el fondo de la raza que aparece en ella. (FOUCAULT, 2014, p. 65)

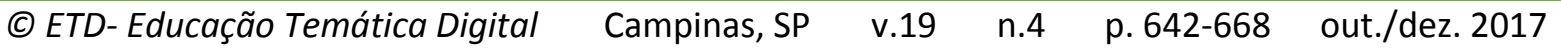


La noción de raza que utiliza Foucault es en un sentido genérico, un concepto que no tiene que ver con la noción moderna de racismo, sino es un modo de diferenciar entre grupos de una misma población, por ejemplo los que pertenecen a tal región, los de la montaña y la costa, los de un lado del rio y los del otro, entre los ricos y los pobres, entre los normales y los anormales, entre los disciplinados y los incorregibles, entre los sanos y los enfermos. Foucault ejemplifica históricamente estas disputas entre normandos, romanos, galos y sajones, etcétera.

La escuela como fábrica de sujetos no sólo educa para la guerra, también educa para el racismo. Es decir, se educa para soportar las relaciones de fuerza que se establecen en toda relación social y para distinguir las razas que conforman a la sociedad en su conjunto. Establecer estas diferencias raciales sostiene la vida de la sociedad moderna. Dicha distinción puede o no considerar elementos fisiológicos para justificar la superioridad e inferioridad habidas al interior de la sociedad. La escuela no sólo reconoce sino que también mantiene, reproduce, elimina y crea estas diferencias tal y como se verá en los ejemplos escolares mexicanos expuestos en el siguiente apartado.

\section{LA ESCUELA EN LAS SOCIEDADES DISCIPLINARIAS Y DE NORMALIZACIÓN}

La guerra de razas es algo que la sociedad va a ejercer sobre sí misma. Las relaciones de poder están atravesadas por un racismo interno que lleva a la purificación permanente, lo cual es, siguiendo a Foucault (2014), una de las dimensiones fundamentales de la normalización social, es decir, la eliminación y la segregación racial están en el nacimiento de las sociedades de normalización. Podemos decir que toda sociedad normalizada se establece bajo principios de purificación. Por ejemplo, en el reconocimiento de los gremios magisteriales en el México virreinal, se comenzó a aglutinar en torno a la escuela a los diversos sujetos por su raza. Ser maestro dejó de ser algo privado y pasó al orden de lo público, de pronto los preceptores abandonaron su condición de "inclasificables" y entraron a las distinciones de razas, se establecieron proclamas, reglas que lo situaban como sujeto distante y distinto a otro, al interior de la propia sociedad del México virreinal.

De acuerdo con Tanck

En 1709 se proclamó en México la importancia y dignidad de la profesión magisterial haciendo explícito los privilegios en las 'Preminencias y leyes de los maestros examinados en la Nobilísima Arte de Leer, Escribir y Contar y Enseñar niños'. 
Podrían los preceptores llevar armas defensivas y ofensivas, traer cuatro lacayos o esclavos con espadas.

'Gozarían todos y qualesquiera preminencia y franquicias de que gozan los hijosdalgos'. Se prohibió al poder público encarcelar a un maestro.

Para ser preceptor el aspirante tenía que probar que era de sangre pura, cristiano viejo, hijo legítimo y de buena vida y costumbres (TANCK, 1984, p. 92)

Asimismo las autoridades del gremio "Pidieron y recibieron el permiso del Virrey para hacer vigente el segundo artículo que decía 'Que el que hubiere de ser maestro no ha de ser negro, ni mulato, ni indio, y siendo español ha de dar información de cristiano viejo, de vida y costumbres" (Tanck, 1984, p. 93). Como puede observarse en las citas anteriores, la raza permite regular y normar la vida de los individuos, la distinción que se hace entre quienes pueden ser o no preceptores no corresponde a lo que hoy entendemos como segregación racial o racismo. No se niega la posibilidad de ser maestro a los de "color quebrado" por su origen de raza sino más bien por el orden social al que pertenecen. Se anuncia lo que, como decíamos, se transformará en la lucha de clases de principios del siglo XIX.

Se es preceptor, en principio, no porque se sepa leer y escribir, cosa de por sí necesaria, sino que se puede enseñar porque se cumple con el criterio racial instituido que ordena a la sociedad. Es a partir de la noción de casta, equivalente a la de raza, que se articula y determina el lugar correspondiente a cada sujeto según la tabla de clasificación que a continuación reproducimos: 


\section{Clasificación de castas en México Virreinal}

\begin{tabular}{|c|c|c|}
\hline \multicolumn{2}{|c|}{ De la unión entre } & Nace \\
\hline Español & Española & Criollo \\
\hline Español & India & Mestizo o Coyote \\
\hline Mestizo & Española & Castizo \\
\hline Castizo & Española & Español \\
\hline Español & Negra & Mulato \\
\hline Mulato & Española & Morisco \\
\hline Salta atrás o torna atrás ${ }^{3}$ & India & Chino \\
\hline Chino & Mulata & Lobo \\
\hline Lobo & Mulata & Gíbaro \\
\hline Gíbaro & India & Albarrazado \\
\hline Albarrazado & Negra & Cambujo \\
\hline Cambujo & India & Zambo o Zambaygo \\
\hline Negro & India & Zambo o Zambaygo \\
\hline Negro & Zamba & Zambo-prieto \\
\hline Zambo & Mulata & Calpán-mulato \\
\hline Calpán-mulato & Zamba & Tente en el aire \\
\hline Tente en el aire & Mulata & No te entiendo \\
\hline No te entiendo & India & Ahí te estás \\
\hline
\end{tabular}

Fuente : México a través de los siglos, 1987, p. 16

Las razas sirven para clasificar, jerarquizar y regular a los individuos que conforman la sociedad moderna. Será a partir de esta regulación racial que los cuerpos se disciplinarán y las sociedades se normarán.

La normalización es un poder regulativo que forma tanto a la individualidad, a través de la disciplina, como a la sociedad, a través de la biopolítica. La disciplina controla los cuerpos, la biopolítica controla las poblaciones, y ambas están atravesadas por la normalización. En otras palabras, la normalización es un ejercicio de poder que regula la vida a través de la disciplina de los cuerpos y el control de la sociedad, por ello la normalización es una forma de poder sobre la vida y de la vida. Un poder que se caracteriza por tener como finalidad la purificación al interior de la sociedad.

\footnotetext{
${ }^{3}$ El salta atrás era el que tenía caracteres de negro, naciendo de familia no negra. Del salta-atrás o torna-
} atrás casado con india nacía el chino que nada tenía que ver con la raza asiática, por supuesto.

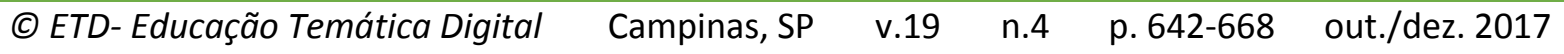


Recordemos que Foucault analiza las nociones de "lucha de razas", "sociedades disciplinarias", "sociedades de normalización" y "biopolítica" principalmente desde la segunda mitad del siglo XVIII. Lo cual es importante porque estos ejercicios de poder nacen en un periodo en el que las ciencias, sobre todo la medicina y el derecho, tendrán una incidencia sustantiva en la vida social, y la escuela será un lugar privilegiado para la justificación y mantenimiento de esta forma de vida.

La escuela en las sociedades disciplinarias

De acuerdo con Foucault, la disciplina es una anatomía política del detalle, dado que se ejerce control hasta en el más mínimo o insignificante aspecto del cuerpo. Gestos, actitudes, rapidez, eficacia y organización de los movimientos, fuerza, ejercicios o procesos de las actividades son formas de gobierno sobre el cuerpo. En otras palabras la disciplina son los métodos:

[...] que permiten el control minucioso de las operaciones del cuerpo, que garantizan la sujeción constante de sus fuerzas y les imponen una relación de docilidad-utilidad es a lo que se puede llamar 'disciplinas' [...] estas disciplinas han llegado a ser, en el transcurso de los siglos XVII y XVIII, fórmulas generales de dominación (FOUCAULT, 2015, p.159)

Estas fórmulas de gobierno son procedimientos, métodos o prácticas concretas que buscan controlar los cuerpos. En el cuerpo se establece la relación entre docilidadutilidad y obediencia-producción. En las sociedades, del siglo XVII y XVIII, la disciplina aumenta las fuerzas del cuerpo, en términos de utilidad económica, y disminuye esas mismas fuerzas, en términos de docilidad y obediencia política. Así, el control del cuerpo se convierte en una aptitud o capacidad deseable. Entre más disciplinado más aptitud económica y política, desde la segunda mitad del siglo XVIII el sujeto se convierte en algo que se fabrica porque se puede maquinizar su cuerpo. De acuerdo con Foucault, la disciplina fabrica sujetos a partir de los cuerpos que controla. Lo cual no quiere decir que Foucault, otorgue un valor negativo, malo o terrible a la disciplina, sino que la identifica como una cualidad sustantiva de la vida de las sociedades modernas. En esta época moderna la educación disciplina al cuerpo de acuerdo con las nuevas necesidades de cierta higiene, tiempo y espacio escolar, asociados con los propósitos específicos del saber moderno o, quizás valga decir, científico-ilustrado.

La escuela de las sociedades disciplinarias no será más un lugar cualquiera, tendrá aire, luz, dimensiones, personas y objetos dispuestos de cierto modo y no de otro. Por ejemplo, 
Cuando se fundó la Compañía lancasteriana en México sus miembros proyectaron una escuela para 1386 niños con un solo profesor. El arquitecto Antonio Villarel presentó un croquis arquitectónico que mostraba cómo iba a acomodar en el ex convento de Belén dicha escuela. Los alumnos estarían ubicados en tres corredores que formaban una " $\mathrm{T}$ "; 660 niños de primeras letras en el salón-corredor más largo; 418 en las escuelas de estudios avanzados en otro corredor; y 308 normalistas en el tercer corredor. (Archivo General de la Nación, en TANCK, 1973, 498)

La ubicación de los cuerpos en el espacio, la distribución de los niños en el edificio y el aula, es una forma de gobierno sobre los cuerpos. La relación entre cuerpos - espacios se establece con precisión científica y tiene como objetivo la higiene escolar. Los conocimientos arquitectónicos están al servicio de principios médicos y de salud.

De pronto el maestro ya no sólo lo es por su raza, sino también por su función vigilante, el alumno es concebido en su puerilidad por las características propias de su desarrollo biológico y social, y el saber se concibe desde su objetividad y cientificidad. Se pasa del súbdito al niño, del preceptor de cierta raza al maestro que vigila y transmite saberes objetivos, de la instrucción doctrinaria a la didáctica científica, y del claustro al aula. El maestro se profesionaliza según cierto saber pedagógico, un saber adscrito a una disciplina y ciencia. El maestro se reconoce en el alumno tanto como el médico lo hace en el enfermo.

Las buenas costumbres no sólo son eso, además son entendidas como higiénicas y socializadoras, se remitirán a sus funciones biológicas, fisiológicas y sociales. La educación en general y la escolarización en particular son un acto público, en tanto ámbito del Estado, son un acto de lo social, de la política y de la ciencia. El control del cuerpo en la escuela está al servicio de una sociedad instrumental y útil. Los cuerpos escolares están sujetos a la relación utilidad-docilidad.

La escuela de las sociedades disciplinarias, inaugura un régimen en donde el discurso sobre la observación y la práctica, sobre la razón y la experiencia, tiene que ver con el nuevo trato que se otorga a los cuerpos; con una disciplina que se puede denominar como militarmente industriosa; con un orden dispuesto a producir sujetos económicamente útiles y políticamente obedientes. Producción de sujetos útiles y obedientes que funda un modo de vida por el cual vale la pena vivir y luchar. 
Siguiendo a Foucault (2015, p. 157-197), identificamos cuatro características en la producción de procesos de subjetivación propias de las sociedades disciplinarias, a saber: distribución de los individuos en el espacio, control de la actividad, organización de las génesis, y composición de fuerzas. No olvidemos que estas características disciplinarias se rigen bajo los principios de guerra y raza, donde el sentido de la disciplina moderna es la purificación. Veamos estas cualidades en la escuela lancasteriana ${ }^{4}$ en México, aquí aparece como tal durante la segunda década del siglo XIX, apenas 20 años después de la publicación de las primeras experiencias en Europa. En 1797 Bell publica su primer libro An experiment in education, y Lancaster en 1803 publica su obra Improvements in education. Aunque es importante decir que en México los Betlemitas ${ }^{5}$ utilizaron la enseñanza mutua en su escuela de primeras letras por lo menos desde 1800. Así lo dice uno de sus alumnos:

[...] éramos trescientos discípulos y cada uno de los más adelantados que se denominaba decurión cuidaba de diez decuriados. Soy muy cobarde para afirmar que antes que nos viniera de Europa el sistema mutuo ya lo usábamos sin darle nombre particular, y acaso sin conocer su utilidad y sus ventajas; tal ha sido nuestro despego a la fama, nuestra ignorancia o nuestro desinterés, que como la tierra deposita en sus entrañas los tesoros que tanto anhelan los hombres sin hacer alarde de lo que encierra, así nosotros poseíamos de tiempo inmemorial un bien sin celebrarlo porque ignorábamos lo que teníamos (Archivo del ex Ayuntamiento de México, en TANCK 1973, p. 495)

\footnotetext{
${ }^{4}$ De manera muy general podemos decir que el método de Lancaster consiste en que los alumnos eran divididos en grupos de diez, quedando en primer lugar el niño más hábil y en décimo lugar el menos capaz. Cada grupo recibía la instrucción de un monitor, que era un niño de más edad y más capacidad, previamente preparado por el director de la escuela. Había ocho grados o clases, que iban desde los niños más pequeños o inhábiles hasta los más grande y hábiles. Se impartían cuatro asignaturas o lecciones que eran las de: escritura, lectura, aritmética y doctrina cristiana. La primera asignatura era la de escritura y los niños, en filas de diez, estaban sentados todos del mismo lado en bancos con mesa y ubicados frente al escritorio del director. Esta primera asignatura se dividía en ocho clases o grados, la primera era para los niños más pequeños y menos hábiles, su mesa tenía una gran cajilla cubierta de arena los niño debían mirar al monitor que dibujaba una letra en la arena y los niños debían delinear sobre ella; en lecciones subsecuentes los niños seguían las instrucciones del monitor que se paraba en un banco al otro lado de la mesa para indicar las letras del alfabeto escritas en un cartón colgado del telégrafo. Los niños de segundo a sexto grado aprendían la escritura en pizarra, el monitor les dictaba palabras de una a cinc sílabas. Los niños de séptimo y octavo grado ejercitaban la escritura en papel y ocupaban las últimas mesas del salón, los de séptimo copiaban escribían trazos copian las letras españolas hechas por Torcuato Torío de la Riva, y los alumnos de octavo copiaban manuscritos y lemas. Al terminar la asignatura de escritura pasaban, sonaba un campana y los niños se levantaban de las mesas e iban a los pasillos a formar grupos semicirculares. En semicírculos tenían la lección de lectura y de doctrina cristiana. La lección de aritmética se tomaba en las mesas, bajo el mismo orden de la lección de escritura. (TANCK, 1973)

${ }^{5}$ Por cierto, se adjudica a los Betlemitas el principio didáctico de "la letra con sangre entra".
}

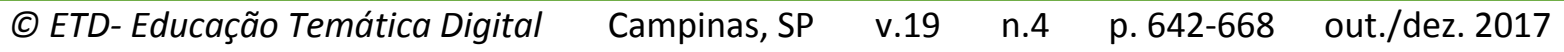


Volviendo a las características disciplinarias; la primera cualidad que se refiere a la distribución de los individuos en el espacio, consiste en que los cuerpos se distribuyen de cierta manera en el espacio, es decir, cierta cantidad de niños en un aula, cierta cantidad de aulas en la escuela, ciertas escuelas en un cuadrante de la ciudad. Además del ejemplo anterior, respecto al acomodo arquitectónico del ex convento de Belén, en la enseñanza mutua los alumnos eran divididos en pequeños grupos de diez; cada grupo recibía la instrucción de un monitor o instructor, que era un niño de más edad y más capacidad, previamente preparado por el director de la escuela.

En fila, de frente al escritorio del maestro, se sucedían, una detrás de otra, largas mesas con bancos de madera para diez alumnos en cada banco. En la primera mesa de cada una de las ocho clases se colocaba un "telégrafo" 6 [...] Cada grupo de diez niños tenía un monitor que, de acuerdo con un horario, enseñaba las lecciones de escritura, lectura, aritmética y doctrina cristiana. Además de estos "monitores particulares" habría "monitores generales" y "monitores de orden". Los monitores generales tomaban la asistencia, averiguaban la razón de la ausencia de un alumno, cuidaban los útiles de la enseñanza y los de orden administraban la disciplina. Todos los monitores eran supervisados por el director de la escuela (GARCíA, 1945 en TANCK, 1973, p. 498-499)

Los cuerpos (los niños) y las cosas (los bancos) son dispuestos y controlados en el espacio del aula, el alumno tiene actividades específicas y organizadas según el espacio y el tiempo del saber pedagogizado.

La segunda característica es el control de las actividades del cuerpo, es decir, en qué momento el alumno debe sentarse, callarse, escribir, levantarse, orar, hincarse, etcétera. Por ejemplo las órdenes de la enseñanza mutua consistían en:

Para hacer cesar la marcha y obtener completo silencio. -Un silbido prolongado. A esta señal todos se detienen, permaneciendo en el silencio más profundo.

Para hacer continuar una marcha interrumpida-. Dice el maestro-: ¡Continuar! Los niños se ponen otra vez en marcha.

Para hacer cesar el estudio de cualquier materia y pasar a otra. Dos silbidos prolongados. Para que los niños saluden cuando visiten la escuela autoridades o personas distinguidas. -Tres silbidos-. Al oír el primero todos los niños suspenden el trabajo: al segundo, si están en los bancos se levantan, si en los semicírculos, se vuelve. Al tercer silbido saluda respetuosamente y guardan la voz de continuar para volver a emprender su ocupación (Larroyo, 1986, p. 233)

\footnotetext{
${ }^{6}$ Este instrumento llamado "telégrafo" consistía en un palo con una placa en la que de un lado aparecían los
} números del uno al ocho que indicaban la clase: y del otro lado las letras "EX" para examen (Tanck, 1984) 
Las señales como órdenes para el control de los movimientos de los niños van forjando sus cuerpos para que sean dóciles y útiles. Vemos funcionar la anatomía política del detalle o disciplina como práctica concreta de dominio corporal.

En tercer lugar está la "organización de las génesis" que consiste en gestionar el espacio y el tiempo de las actividades del individuo desde su origen, de tal manera que esta organización sirva de base para la capitalización del espacio y tiempo social, es decir, dividir el ciclo de la vida en segmentos de escolarización, tiempo para cursar el preescolar, enseguida el nivel básico, luego el medio y finalmente la educación superior. Esto puede verse desde la primera propuesta de Comenio de dividir la escuela conforme a los años de crecimiento, así se tendría la escuela maternal para la infancia, la escuela de letras o escuela común pública para la puericia, la escuela latina o gimnasio para la adolescencia y la Academia para la juventud. A diferencia de lo propuesto en la Didáctica Magna, en la enseñanza mutua, el progreso en las habilidades de los niños era lo que determinaba el grado en el que se encontraba en cada asignatura. Es decir, la organización del tiempo, el espacio y las actividades eran establecidas por las capacidades del niño y no sólo por su edad. Por ejemplo

[...] en la escuela municipal de la Ribera de San Cosme, un niño de 6 años entró en diciembre de 1834 en la primera clase de todas las asignaturas. Once meses después, en noviembre de 1835, se hallaba en el primer lugar de la segunda clase de lectura, todavía en la primera clase de escritura, en la segunda clase de aritmética y en la primera clase de doctrina cristiana y civil. Otro compañero de este niño, teniendo la misma edad y entrando a la escuela al mismo tiempo y en la primera clase de todo, avanzaba en los once meses hasta encontrarse en el tercer lugar de la tercera clase de lectura; en la primera clase, tercera sección de escritura; en la tercera clase de aritmética; tercera de doctrina cristiana y segunda de doctrina civil. Se consideraba que un estudiante podría terminar el curso completo en dieciocho meses, aunque los maestros se quejaban de que pocos niños lo lograban. (Tanck, 1973, p. 504)

Si cada curso duraba 18 meses y son 8 cursos o grados los que había que cursar, podemos decir que el niño estaba en la escuela 144 meses, es decir 12 años. La vida del niño estaba dividida en 8 segmentos de 18 meses cada uno y en conjunto conformaban un segmento mayor que es el de la escolarización que duraba 12 años.

Finalmente, la cuarta característica es la composición de fuerzas, donde se concibe al cuerpo como engranaje de una máquina multisegmentaria. Se trata de extraer la máxima capacidad-aptitud de cada cuerpo para articularlo con otros cuerpos que en su conjunto son la fuerza productiva de la escuela, de la fábrica, del ejército o de la sociedad en su conjunto. Por ejemplo, 
Esta rigurosa disciplina [de la enseñanza mutua] de inspiración entre militar e industrial se acompaña de un sistema continuo de valoración del aprovechamiento y de la conducta [...] la valoración comporta el incesante cambio de lugar de los alumnos en las bancas de su división, o el paso de una división a la otra: [...] si un escolar comete una falta, cede su lugar a aquel situado detrás del que lo corrige, y así el más hábil viene a colocarse en primer lugar [...] El niño que durante algún tiempo ha ocupado el primer lugar de la clase pasa al último lugar de la clase superior. Por otro lado, el que no hace progresos suficientes, desciende al primer lugar de la clase inferior (Manacorda, 1987, p. 408-409)

Es sin duda un riguroso sistema escalafonario, pero que permanece en el terreno de las apariencias, de lo visible, no logra penetrar en la voluntad de los niños, tan sólo los ordena y jerarquiza pero, a pesar de sus limitaciones, logra algunos de los propósitos más importantes de la época, que son: lograr que las personas se instruyeran así mismas, disminuir los gastos en la educación, reducir la necesidad del maestro y atender a las clases populares ${ }^{7}$.

Algunos niños o jóvenes abandonaban la escuela para trabajar y ayudar a sostener a su familia, "salían de la escuela tan pronto como sabían suficiente para ganar su jornal, colocándose los más afortunados ya en el comercio, ya a aprender un oficio, o los más pobres recogiendo palos, boñigas o basura" (Tanck, 1973, p. 511) Esto es claro ejemplo de la inserción del cuerpo escolar en el engranaje de una máquina segmentaria en comercio, servicios, industria de la transformación, etcétera.

Es interesante notar que el método de Lancaster, propio de las sociedades disciplinarias, se acerca a la llamada "educación activa", tan en boga en la segunda mitad del siglo XX, que se caracteriza por la individualización de la enseñanza en la cual cada niño progresa en cada asignatura según su propia velocidad de aprendizaje.

La experiencia lancasteriana no representa solamente un método didáctico, es ante todo una rápida respuesta política y económica, frente al surgimiento de demandas que refieren a la vida y al trabajo. En México la enseñanza mutua, propuesta por Lancaster, fue bien acogida, pues además de constituir una punta de lanza ante el viejo discurso de la dominación española, representaba la posibilidad de constitución de un nuevo sujeto, adecuado a las expectativas de progreso y modernidad, de razón y ciencia, utilidad económica y obediencia política.

\footnotetext{
${ }^{7}$ Efectivamente los niños mexicanos que asistían a la escuela lancasteriana eran de clase popular o mejor dicho eran pobres, muchas veces los niños asistían sin desayunar, no contaban con ropa para salir de su casa e incluso acudían descalzos. Esta condición de pobreza provocaba que alrededor del $50 \%$ de los niños inscritos no asistieran a la escuela (Archivo del ex Ayuntamiento de México, en Tanck, 1973, p. 510)
} 
Sin duda la disciplina existe y es sustantiva en la conformación individual y social desde tiempos inmemoriables, en el mundo antiguo, en el mundo precolombino, entre los antiguos cristianos o en el propio Comenio quien en 1632 en su Didáctica Magna sostiene: "Cierto es aquel proverbio tan repetido y popular entre los bohemios: Escuela sin disciplina es molino sin agua. De igual manera que si quitas el agua a un molino, se parará al momento, si suprimes la disciplina en una escuela, forzosamente han de retardarse todas las cosas" (Comenio, 1986, p. 265). Desde Comenio se puede hablar de las cimientes que darán paso a la disciplina en la escuela moderna, sin embargo, la diferencia está en que las sociedades disciplinarias desde finales del siglo XVIII responden al estado de guerra y a la regulación racial para purificar a la sociedad desde su interior. La disciplina de la escuela moderna se puede denominar como militarmente industriosa. Se disciplina al cuerpo, no para combatir al extranjero, sino para segregar o exterminar al originario nacido al interior de la propia sociedad. Se militariza y mecaniza al cuerpo para hacer que la vida en general sea más sana.

En pocas palabras, seguramente las cuatro características de la disciplina moderna analizadas arriba están en otras prácticas educativas no modernas, sin embargo la diferencia está en las condiciones de guerra-raza-pureza que se establecen en las sociedades de la época clásica. Y la escuela como fábrica de sujetos está marcada por esta guerra de razas que busca sanar, medicalizar, higienizar, purificar a la sociedad a través de la disciplina.

Las sociedades disciplinarias y de normalización no se excluyen antes bien se articulan. La norma se aplica tanto para un cuerpo como para una población. Lo interesante es que estas normas surgirán a partir de un racismo, propio de las sociedades modernas, que pretende purificar a la sociedad desde su interior.

\section{La escuela en las sociedades de normalización}

Ahora veamos cómo esta condición de guerra-raza-pureza se presenta en las sociedades de normalización. La normalización es el ejercicio de poder que se ejecuta con la norma más que con la ley. De acuerdo con Castro (2011) existen tres distinciones entre norma y ley: 1) la norma mide cuantitativamente y jerarquiza en términos de capacidades de los individuos, la ley califica los actos de los individuos como permitidos o prohibidos; 2) la norma siempre busca homogenizar las conductas y los cuerpos, mientras que la ley busca condenarlos o aceptarlos; 3) la norma evidencia el límite entre lo que es exterior a ella, es decir establece las diferencias entre lo que es normal y lo que es anormal, en cambio para la ley las conductas, de anormales o no, siempre son aceptables o 
condenables pero siempre dentro de la ley. Así tenemos que la norma jerarquiza según las capacidades del individuo, homogeniza las conductas y diferencia entre normal y anormal.

Por ejemplo, en 1887, Joaquín Baranda, ministro de Justicia e Instrucción Pública en México, afirmaba en el discurso de inauguración de la Escuela Normal el carácter regulador normativo que debería tener la escuela "matriz" que al mismo tiempo le otorgaba un sentido práctico, de "clínica".

El nombre de Escuela Normal explica bien el objeto de tal institución: sirve de norma y da la regla a que debe ajustarse la enseñanza en la escuela matriz o central de la que se derivan las demás escuelas. En la Normal se forma y educa al maestro perfeccionando sus conocimientos y aprendiendo prácticamente a transmitirlo: haciendo en las escuelas anexas la clínica del profesorado.

Enseñar a enseñar. Este es el programa de las Escuelas Normales. Aquí se formará al maestro: aquí adquirirá los conocimientos y el carácter responsable y bondadoso que exige el ejercicio de sus augustas funciones. El maestro... no es el dómine ignorante y locuaz de que se ha apoderado la caricatura; no es tampoco el tirano inconsciente de la niñez que impone el bárbaro principio de que "la letra con sangre entra". No, ya sabéis lo que debe ser el maestro de una escuela en el siglo XIX. Lo será completamente entre nosotros, cuando ilustrado y enaltecido salga de la Escuela Normal, con un título... para ir a predicar por todos los ámbitos de la República el evangelio de la enseñanza. (Baranda en Martínez, p. 1912)

Con el ejemplo anterior identificamos las tres características de las normas: primero, el maestro normalista ocupa un alto lugar en la jerarquía del dominio y enseñanza de conocimientos, este lugar lo adquiere por sus capacidades; segundo, surge como único capaz de convertir la enseñanza en un método objetivo y científico, digamos pedagogizado, con el cual homogeniza la enseñanza; y finalmente, los normalistas se diferencian de todos aquellos que están fueran de la norma del ser normalista, es decir, se distingue de los ignorantes y tiranos, y se asume como predicador de la enseñanza. El maestro al convertirse en normalista se transforma en el ejecutor de la norma de enseñanza, la Escuela Normal tiene como objetivo normar y homogenizar los procesos de enseñanza, el maestro normalista se ve a sí mismo no como un ser más de la enseñanza, sino como la parte fundamental, es el ordenador y homogeneizador del proceso educativo escolar (Jiménez, 1992). Si bien es cierto muchos pueden ser maestros pero no todos pertenecen al magisterio, desde entonces en México esa condición es exclusiva de los maestros normalistas.

Como hemos señalado "la norma es lo que puede aplicarse tanto a un cuerpo al que se quiere disciplinar como a una población a la que se pretender regularizar [...] La sociedad de normalización es una sociedad donde se cruzan, según una articulación ortogonal, la norma de la disciplina y la norma de la regulación" (FOUCAULT, 2014, p. 228-

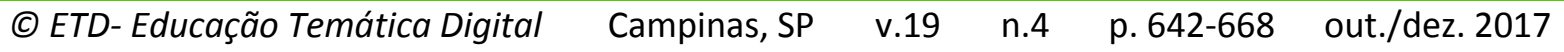


229) Las sociedades de normalización controlan tanto la vida de los cuerpos individuales como a las poblaciones en su conjunto; no son una superación de las disciplinarias, antes bien, lo que se enfatiza en las primeras es el ejercicio de poder que se tiene sobre las poblaciones y en las segundas el que se ejerce sobre los cuerpos. Las escuelas normales no sólo se dirigen a disciplinar los cuerpos individuales de los profesores, sino también normalizan a la sociedad entera a través de la educación. Dichas escuelas se nombran como normales y no disciplinarias.

Por ejemplo en 1854 se decretó el Plan General de Estudios que normaba (no legalizaba) con detalle la educación primaria, secundaria y hasta los estudios superiores profesionales y especiales, el decreto consta de quince títulos, el décimo título

[...] trata del profesorado, que constituye una carrera distinguida, cuyos méritos serán considerados para otros destinos en los diversos ramos de la administración pública. Goza de las siguientes prerrogativas: estar exento del servicio militar y de todo cargo concejil. Además no ser detenido en cárceles públicas. De las mismas prerrogativas disfrutará el inspector de instrucción pública, los rectores o directores y los individuos del consejo de instrucción pública. Para ser catedrático de secundaria se requiere ser mexicano por nacimiento o naturalización, tener 22 años cumplidos, buena conducta y ser bachiller en filosofía (Meneses, 1998, p. 164)

Sumado a esto en 1885 con el nacimiento de la Escuela Modelo de Orizaba, Castellanos dice "el secreto está en la educación de las masas populares y el factor principal en las escuelas normales; pero ¿qué entiendo por escuela normal? [...] lo que caracteriza la escuela normal es la aplicación teórico práctica de la doctrina... como tal, debe ser científica y debe ser práctica" (en Solana, 1981, p. 430). Tanto en la normal de Orizaba de 1885 como en el Plan General de Estudios de 1854 se busca disciplinar el cuerpo del normalista, éste debe tener una carrea distinguida, contar con 22 años, tener buena conducta y ser capaz de aplicar la ciencia y practicar su enseñanza; y al mismo tiempo la normal pretende regularizar a la población, su función es educar a las masas y considerar al profesorado como un gremio distinguido.

También las normas del sistema médico se reflejan en el detalle de las prácticas y principios de higiene escolar, toda la organización escolar está atravesada por una norma higiénica y por la mirada de la medicina social. Por ejemplo en las escuelas lancasterianas de la ciudad de México del siglo XIX, el niño al entrar a la escuela en la mañana "se formaba en línea con sus compañeros de clase para la inspección de cara, manos y uñas: su ropa debe estar limpia, sus zapatos o sus pies sin lodo" (Tanck, 1973, p. 500) La higiene escolar busca preservar la salud del cuerpo y del alma de los niños garantizando a estos que "al poner su delicada planta en el dintel de la escuela, sepan con seguridad que allí

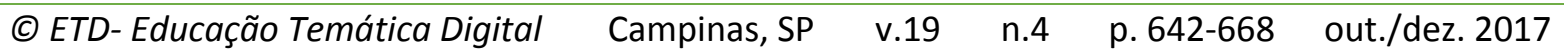


dentro están los tesoros de su engrandecimiento del cuerpo y alma, a la vez que la higiene guardará incólume el tesoro de la salud"' (Ruiz, 1900, p. 199)

Las normas médicas controlan incluso la construcción interna de las escuelas, así puede verse en el Congreso Higiénico Pedagógico de 1882 en México

[...] la figura de las salas no es indiferente: hay que indicar la forma paralelográmica, remplazando los ángulos por superficies cóncavas. Esta disposición es la más propicia, así para la higiene porque facilita la ventilación, como para la acústica, pues favorece mejor las condiciones de la voz [...] El aire atmosférico contiene 20.9 por ciento de oxígeno, el aire espirado no contiene sino 16.03. La respiración ha quitado al ambiente 4.87 por ciento de oxígeno y esto 16 veces más por minuto, y ha vertido en igual tiempo 4.34 en volumen de ácido carbónico, el vapor de agua, y otros productos no bien determinados, procedentes de los cambio entre medio exterior e interior del organismo, son los cuerpos que producen la viciación del aire confinado. (Memorias del Primer Congreso Higiénico Pedagógico de 1882, 1883, p. 34-40)

Toda intención por establecer hasta las hendiduras más mínimas, una razón fundada en las normas médicas, es característica fundamental de las sociedades de normalización que buscan regular la vida de los cuerpos y de la sociedad. Las condiciones de ventilación e iluminación se encuentran perfectamente prescritas sobre bases médicas, estadísticas y físico-químicas. En los congresos higiénico pedagógicos se establecieron una a una las prescripciones que daba cuenta de la construcción y disposición de las escuelas, desde los detalles más ínfimos, como poner perillas en los barandales de las escaleras para que los niños no se resbalaran sobre ellos, hasta los más generales, como la distancia entre cada escuela y el número de ellas por habitantes.

Un ejemplo más de la articulación entre las normas disciplinarias y las de regulación es el siguiente, el Reglamento de las Condiciones Generales de Trabajo del Personal de la Secretaría de Educación Pública de México, Publicado en el Diario Oficial de la Federación el 29 de enero de 1946 con vigencia actual, refiere en diversos artículos a las condiciones de salud que deben tener los trabajadores de la Secretaría de Educación Pública.

Artículo 12- Para formar parte del personal de la Secretaría, se requiere:

I. Tener por lo menos 16 años cumplidos.

II. Presentar una solicitud utilizando la forma oficial que autorice la Secretaría; dicha forma deberá contener los datos necesarios para conocer los antecedentes del solicitante y sus condiciones personales.

III. Ser de nacionalidad mexicana, con la salvedad prevista en el artículo 60 del Estatuto Jurídico.

IV. Estar en el ejercicio de los derechos civiles y políticos que le corresponda, de acuerdo con su sexo y edad.

V. Gozar de buena reputación y no haber sido condenado por delitos graves. 
VI. No haber sido separado de algún empleo, cargo o comisión por motivos análogos a los que se consideran como causas de destitución, a no ser que, por el tiempo transcurrido que no será menor de dos años a partir de su separación, la Secretaría estime que son de aceptarse sus servicios.

VII. No tener impedimento físico para el trabajo, lo que se comprobará con el examen médico en la forma prevista por este Reglamento.

VIII. Tener los conocimientos necesarios para el desempeño del cargo, a juicio del Jefe de la dependencia donde exista la vacante, o sujetarse al concurso o pruebas de competencia que fije la Secretaría. En caso de empleo técnico, acreditar la posesión del título profesional registrado.

IX. Rendir la protesta de ley.

X. Caucionar su manejo, en su caso.

XI. Tomar posesión del cargo.

(Reglamento de las Condiciones Generales de Trabajo del Personal, 1946, p. 4)

Están en el mismo nivel de control disciplinario y de regulación: la edad, la solicitud oficial de ingreso, la nacionalidad, los derechos civiles y políticos, la reputación, la garantía de no haber sido expulsado de un cargo, el estado físico (salud), los conocimientos, la protesta de ley, la garantía de cumplir con el contrato y la asunción del cargo. Sólo los que cumplan con estas normativas pueden pertenecer al personal de la Secretaría de Educación Pública. Las normas siempre buscarán ser la medida de las capacidades de los individuos y de las poblaciones, pretenderán homogenizar los cuerpos y las sociedades, intentarán diferenciar a los individuos normales de los anormales al interior de la sociedad; no olvidemos que esta distinción tiene como finalidad la purificación y la sanación social.

Siguiendo a Foucault, en las sociedades de normalización las reglas del sistema médico se insertan de manera explícita en el sistema jurídico, "la medicina moderna no es una medicina individual o individualista, sino que es una medicina social cuyo fundamento es una cierta tecnología del cuerpo social; la medicina es una práctica social, y sólo en alguno de sus aspectos es individualista y valora las relaciones entre el médico y el paciente" (FOUCAULT, 1999, p. 365) Del mismo modo la educación moderna siempre será pública y social aunque el financiamiento sea privado. El vínculo ortogonal de la norma disciplinaria y la norma de regulación es evidente en las normas del sistema médico. Es decir, los expedientes de los enfermos indican particularidades de la enfermedad de su cuerpo vinculados con la salud de la sociedad.

El control de las normas médicas se ejerce no sólo en el cuerpo individual sino, sobre todo, en el cuerpo social, lo que se controla es la vida de las poblaciones, así, desde inicios del siglo XIX, la medicina es una estrategia biopolítica. Lo mismo sucede con el control de las normas educativas, éstas no sólo se ejercen sobre un cuerpo individual sino sobre todo el cuerpo social, lo que la educación controla es la vida de las poblaciones. La 
biopolítica que nace en el siglo XVIII cuando el dominio de la vida de las poblaciones está en función del "hacer vivir y dejar morir" (FOUCAULT, 2014, p. 223). Un "hacer vivir" que implica el nacimiento de la estadística, este saber técnico-científico del Estado, que justifica, procura y asegura la vida como natalidad, mortalidad, higiene, alfabetización, alimentación, etcétera. Digamos que el cuerpo humano individual, desde sus condiciones de "salud", se reconoce y se inserta política y socialmente como fuerza de trabajo y como cuerpo productivo.

En el ejemplo anterior vemos que el reglamento para formar parte de la Secretaría establece como requisito "no tener impedimento físico para el trabajo, lo que se comprobará con el examen médico en la forma prevista por este Reglamento". Esta norma, como todas las que se incluyen en el citado Reglamento, afecta a maestros, burócratas, personal de intendencia, técnicos, etcétera., es decir, a todo trabajador de la Secretaría de Educación Pública. El maestro es disciplinado y regulado, ya no como poseedor de la teoría y la práctica de la enseñanza, sino como trabajador al servicio del Estado. La relación de maestro ya no está establecida al soberano, sino con relación al Estado.

Una vez que el trabajador, maestro o no, ingresa a la Secretaría de acuerdo con el artículo 25 tiene la obligación de "en caso de enfermedad dar el aviso correspondiente a la dependencia de su adscripción y al servicio médico, dentro de la hora siguiente a la reglamentaria de entrada a sus labores, precisando el lugar en que deba practicarse el examen médico" (Reglamento de las Condiciones Generales de Trabajo del Personal, 1946, p. 4).

En el cruce entre normas disciplinarias y de regulación hay una relación directa entre cuerpo, salud y fuerza productiva de los individuos. No se trata de la salud del cuerpo individual, que está más relacionado con la disciplina, sino que se trata de la salubridad, es decir del control de la "salud" de la población. En las sociedades de normalización la salud de los cuerpos de los profesores normalista, como los de cualquier trabajador del Estado, está determinada por el sistema de salubridad. La enfermedad es tratada como problema de utilidad económica, por ello el cuerpo enfermo, incluyendo el del maestro normalista, se administra desde la relación trabajo-salario. Los cuerpos y las prácticas de la enseñanza propia de los maestros normalistas desaparecen en lo individual al insertarse en la nómina laboral.

Con las tres distinciones entre norma y ley, que señalamos al inicio de este apartado, vemos que el sistema jurídico está más vinculado con el sistema médico. Dicho sistema se ha centrado en la normalización tanto de los cuerpos como de las poblaciones

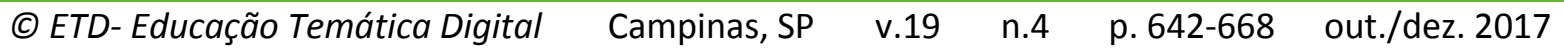


ya que ha distinguido entre los normales y los anormales, identificando y clasificando la salud y la enfermedad. Podemos decir que el sistema médico tiene autoridad no sólo sobre la salud y enfermedad de cuerpos y poblaciones, sino sobre la vida misma.

La normalización y la disciplina cuidan los más mínimos detalles del control de los cuerpos colectivos e individuales. La disciplina, con la anatomía política del detalle que fabrica sujetos útiles económicamente y dóciles políticamente, y la normalización, que jerarquiza según las capacidades del individuo, homogeniza las conductas y diferencia entre normal y anormal, son formas de control de la vida y sobre la vida que posibilitan procesos de subjetivación. En las sociedades disciplinarias y de normalización de la época clásica hay un vínculo inseparable entre guerra, raza y purificación. La medicina social se inserta hasta en el más mínimo detalle de la vida individual y colectiva. Por la salud de la sociedad es necesaria la guerra de razas al interior de cada población.

\section{REFLEXIONES FINALES}

Como se visto en las prácticas escolares se establecen principios de saneamiento a través del control del cuerpo y de la sociedad. La regulación del magisterio a partir de su raza, la higiene escolar, el trabajo del cuerpo al servicio del Estado, son muestras de la serie guerra-raza-purificación inmersa en la escuela. La normal representa para la historia de la escuela lo que la clínica para la historia del hospital. Tanto la clínica como la normal norman y normalizan a los cuerpos individuales como a las poblaciones en su conjunto.

En el siglo XVIII en México ser maestro significaba corresponder estrictamente al orden de la raza del virreinato, es decir, "el que hubiere de ser maestro no ha de ser negro, ni mulato, ni indio, y que siendo español ha de dar información de cristiano viejo, de vida y costumbre". En el siglo XIX se requería vincular la profesión de maestro a un saber normalizado que en principio servía de norma para regularizar la enseñanza "el maestro normalista debe predicar el evangelio de la enseñanza y aplicar teórica y prácticamente los saberes científicos de dicha doctrina". Y en el siglo XX y lo que va del XXI el maestro normalista es, en principio y fundamentalmente, un trabajador medicalizado al servicio del Estado, ya que "no debe tener impedimento físico para el trabajo lo que se comprobará con el examen médico correspondiente al Reglamento".

Lo que ocurre con los cuerpos de la enseñanza se circunscribe al biopoder ya sea como disciplina, normalización o biopolítica, es decir, el control de la vida individual y colectiva es constitutivo de las prácticas docentes y sus saberes pedagogizados. 
La escolarización en México del siglo XVIII fabricaba subjetividad a través de la distinción racial como conjunto de cualidades visiblemente diferentes que permitieron articular y clasificar a los sujetos. Conjunto de sujetos producido por la guerra, por los ejercicios de poder, que es, como señala Foucault, el motor de las instituciones y el orden. La lucha de clases propia del siglo XIX, derivada de la lucha de razas, es fundamento de las sociedades de normalización, las escuelas normales son vivo ejemplo de los procesos de clasificación, segregación y exclusión. Procesos que permitieron vivir de algún modo, estamos, como dice Foucault, destinados y sometidos a cierta manera de vivir y a cierta manera de morir, en función de discursos verdaderos que llevan consigo efectos específicos de poder.

Desde el siglo XVIII la escuela como fábrica de sujetos forma parte de la defensa de la sociedad moderna, ya sea disciplinaria o de normalización, que no es otra cosa que el control de la vida misma. Toda defensa de la sociedad son formas de ejercicios de poder que gobiernan la vida, y toda práctica escolar, desde el siglo XVIII, es una manera de defender la sociedad. El poder lo que quiere es mantenerse a sí mismo para sostener la vida. Las prácticas educativas disciplinarias y de normalización tratan del poder que se ejerce sobre la vida y simultáneamente del poder de la vida.

La escuela moderna es el lugar donde el imperativo "Hay que defender la sociedad" es evidente, ya que las prácticas escolares asumen que no hay sujeto ni institución neutral y tampoco hay ejercicio de poder imparcial, asumen que los ejercicios de poder son el motor de la producción de subjetividad; y que la "sociedad" es la noción que da sentido al grupo o a la comunidad a la cual se pertenece, y por la cual se nos "hace vivir" a costa incluso de la vida de otras sociedades o formas de vida.

Defender la escuela es defender las prácticas disciplinarias y de normalización que fabrican sujetos. En la escuela se observan las dos series que Foucault señala a propósito de la disciplina y la biopolítica "la serie cuerpo-organismo-disciplina-instituciones; y la serie población-procesos biológicos-mecanismos regularizadores" (FOUCAULT, 2014, p. 226). Ambas series son manifestaciones del control de la vida, la primera como dominio del cuerpo a través de la disciplina, y la segunda como el gobierno de las poblaciones por medio de la biopolítica, ambas están atravesadas por el poder normativo.

Lo que Foucault llama sociedades de seguridad son justamente estas sociedades que de manera descarnada garantizan la vida, "hacen vivir" a toda costa, incluso en nombre "de la vida" se mata, se masacran sociedades enteras. Por ello la normalización como control de la vida, "es una forma de biopoder que aparece cuando el hombre tiene técnica y políticamente la posibilidad no sólo de disponer la vida sino de hacerla proliferar,

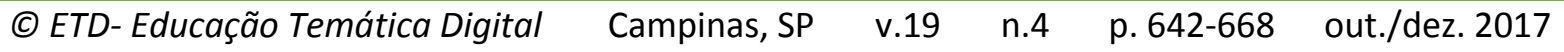


de fabricar lo vivo, lo monstruoso y, en el límite virus incontrolables y universalmente destructores" (FOUCAULT, 2014, p. 229)

Este ensayo pretende ser un ejercicio crítico en torno a la escuela como fábrica de sujetos útiles, dóciles y sanos. La crítica, como la libertad, no es indisciplina ni negación del sometimiento, más bien la crítica es un ejercicio de poder que se empeña en evidenciar el mal habido en el bien, la guerra habida en la paz, la diferencia habida en la identidad, la barbarie habida en la educación, la exclusión habida en la inclusión. La crítica convierte la frase: "no hay mal que por bien no venga" en "no hay bien que por mal no venga". Tampoco hay salud que por enfermedad no venga, ni conocimiento que por ignorancia no venga como no hay civilización que por barbarie no venga.

\section{REFERENCIAS}

CASTRO, Edgardo. Diccionario Foucault. Temas, conceptos y autores. Buenos Aires: Siglo XXI, 2011.

MEMORIAS del Congreso Higiénico Pedagógico de 1882, México: Imprenta del Gobierno, en Palacio, 1883.

COMENIO, Juan Amós. Didáctica magna. Madrid: Akal, 1986.

FOUCAULT, Michel. Defender la sociedad. México, DF: Fondo de Cultura Económica, 2014.

Vigilar y castigar. Nacimiento de la prisión. México, DF: Siglo XXI, 2015.

Estrategias de poder. México, DF: Paidós, 1999.

JIMÉNEZ, Marco. Los cuerpos y las cosas de la enseñanza. Tesis de maestría en ciencias con especialidad en educación México: DIE-CINVESTAV-IPN, 1992.

LARROYO, Francisco. Historia comparada de la educación en México. México, DF: Porrúa, 1986.

MANACORDA, Mario. Historia de la educación (Tomo I y II). México, DF: Siglo XXI, 1988.

MARTÍNEZ, M. “Discurso pronunciado en la celebración del XXV aniversario de la Escuela Nacional de Maestros (bodas de plata)", en Archivo Histórico de la SEP. 29-7-32-13, México, DF, 1912.

MENESES, Ernesto. Tendencias educativas oficiales en México. 1821-1911. La problemática de la educación mexicana en el siglo XIX y principios del siglo XX. México, DF: Universidad Iberoamericana, 1998.

MÉXICO a través de los siglos. Tomo VI. México, DF: Ed Cumbre, 1987.

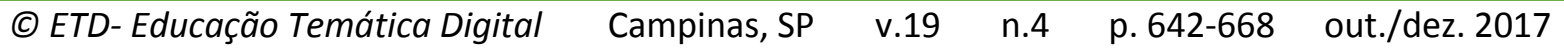


REGLAMENTO de las Condiciones Generales de Trabajo del Personal de la Secretaría de Educación Pública. México, DF: SEP, 1946.

SOLANA, Fernando, CARDIEL, Raúl y BOLAÑOS, Raúl. Historia de la educación pública en México. México: Fondo de Cultura Económica, 1981.

TANCK, Dorothy. "Las escuelas lancasterianas en la Ciudad de México: 1822-1842", Historia Mexicana. Vol 22, №. 4, Ensayos sobre la historia de la educación en México (apr./ jun., 1973), pp. 494-513, 1973.

La educación ilustrada. México, DF: Colegio de México, 1984.

${ }^{i}$ Revisão gramatical do texto sob a responsabilidade dos autores 